\title{
A new collocation method for solution of mixed linear Integro-differential difference equations
}

\author{
Berna Bulbul Aslan ${ }^{1}$, Burcu Gurbuz ${ }^{2}$ and Mehmet Sezer ${ }^{3}$ \\ ${ }^{1}$ Department of Mathematics, Faculty of Science, Mugla Sitki Kocman University, Mugla, Turkey \\ ${ }^{2,3}$ Department of Mathematics, Faculty of Science, Celal Bayar University, Manisa, Turkey
}

Received: 4 February 2015, Revised: 6 February 2015, Accepted: 20 February 2015

Published online: 23 February 2015

\begin{abstract}
The aim of this article is to present an efficient numerical procedure for solving mixed linear integro-differential-difference equations. Our method depends mainly on a Taylor expansion approach. This method transforms mixed linear integro-differentialdifference equations and the given conditions into matrix equation which corresponds to a system of linear algebraic equation. The reliability and effiency of the proposed scheme are demonstrated by some numerical experiments and performed on the computer program in Maple10.
\end{abstract}

Keywords: Mixed linear integro-differential-difference equations; Taylor polynomials and series; collocation points.

\section{Introduction}

Since beginning of $1994 \mathrm{~s}$ Taylor matrix and Taylor collocation methods to solve linear differential, integral, integro-differential, difference, integro-difference and systems of integro differential equations have been used by Sezer et al. [1-10]. Our purpose in this study to develop and apply the mentioned methods above to mixed linear integro-differential-difference equation which is given in [11]. These equations are encountered in various fields of science and numerous applications (in elasticity, plasticity, heat and mass transfer, oscillation theory, fluid dynamics, biomechanics, game theory, control, queuing theory, electrical engineering, economics, medicine, etc.)

Consider the mixed linear integro-differential-difference equation:

$$
\sum_{i=0}^{m} p_{i}(x) y^{(i)}(x)+\sum_{i=0}^{m} \sum_{j=0}^{n} q_{i j}(x) y^{(i)}\left(\eta_{i j} x+\tau_{i j}\right)+\sum_{j=0}^{n} \int_{a}^{b} K_{j}(x, t) y^{(j)}(x-t) d t=g(x)
$$

under the mixed conditions

$$
\sum_{j=0}^{m-1}\left[a_{i j} y^{(j)}(a)+b_{i j} y^{(j)}(b)+c_{i j} y^{(j)}(c)\right]=\mu_{i}, \quad i=0,1,2, \ldots, m-1, a \leq b \leq c
$$

where $p_{i}(x), q_{i j}(x)$ and $K_{j}(x, t)(i=0,1, \ldots, m ; j=0,1, \ldots, n)$ are functions that have derivatives on an interval $a \leq x, t \leq b$, and also $a_{i j}, b_{i j}, c_{i j}$ and,$\mu_{i}$ are suitable constants. We consider the solution of (1) in the form

$$
y(x)=\sum_{n=0}^{N} y_{n}(x-c)^{n}, y_{n}=\frac{y^{(n)}(c)}{n !}, a \leq x, c \leq b
$$

\footnotetext{
*Corresponding author e-mail: burcu.gurbuz@cbu.edu.tr
} 
which is the truncated Taylor series of degree $N$ near $x=c$; where $y_{n}, n=0,1, \ldots, N$ are the coefficients to be determined. In addition, to compute the Taylor coefficients we use collocation points defined by

$$
x_{s}=a+\frac{b-a}{N} s, s=0,1, \ldots, N, a=x_{0}<x_{1}<\ldots<x_{N}=b
$$

\section{Fundamental matrix relations}

Let us first write Eq.(1) in the

$$
D(x)+F(x)+I(x)=g(x)
$$

where

$$
\begin{gathered}
D(x)=\sum_{i=0}^{m} p_{i}(x) y^{(i)}(x), F(x)=\sum_{i=0}^{m} \sum_{j=0}^{n} q_{i j}(x) y^{(i)}\left(\eta_{i j} x+\tau_{i j}\right), \\
I(x)=\sum_{j=0}^{n} \int_{a}^{b} K_{j}(x, t) y^{(j)}(x-t) d t
\end{gathered}
$$

Let us consider the Eq.(1) and find the matrix forms of each term of the equation. We first consider the solution $y(x)$ defined by a truncated series (3). Then we can put series (3) in the matrix

$$
[y(x)]=\mathbf{X}(x) \mathbf{Y}
$$

where

$$
\begin{aligned}
& \mathbf{X}(x)=\left[1(x-c)(x-c)^{2} \cdots(x-c)^{N}\right] \\
& \mathbf{Y}=\left[\begin{array}{lllll}
y_{0} & y_{1} & y_{2} & \cdots & y_{N}
\end{array}\right]^{T}
\end{aligned}
$$

On the other hand the relation between the matrix $\mathbf{X}(x)$ and its derivative $\mathbf{X}^{\prime}(x)$ is

$$
\mathbf{X}^{\prime}(x)=\mathbf{X}(x) \mathbf{B}
$$

where

$$
\mathbf{B}=\left[\begin{array}{ccccc}
0 & 1 & 0 & \cdots & 0 \\
0 & 0 & 2 & \cdots & 0 \\
\vdots & \vdots & \vdots & \ddots & \vdots \\
0 & 0 & 0 & \cdots & N \\
0 & 0 & 0 & \cdots & 0
\end{array}\right]
$$

Using the relations (6) and (7) we have recurrence relation

$$
\begin{gathered}
{\left[y^{\prime}(x)\right]=\mathbf{X}^{\prime}(x) \mathbf{Y}=\mathbf{X}(x) \mathbf{B Y}} \\
{\left[y^{\prime \prime}(x)\right]=\mathbf{X}^{\prime}(x) \mathbf{B} \mathbf{Y}=\mathbf{X}(x) \mathbf{B}^{2} \mathbf{Y}} \\
\vdots \quad \vdots \quad
\end{gathered}
$$




$$
\left[y^{(k)}(x)\right]=\mathbf{X}^{\prime}(x) \mathbf{B}^{(k-1)} \mathbf{Y}=\mathbf{X}(x) \mathbf{B}^{k} \mathbf{Y}
$$

By putting $x \rightarrow \lambda x+\eta$ in the relation (6) we obtain the matrix form

$$
[y(\lambda x+\eta)]=\mathbf{X}(\lambda x+\eta)+\mathbf{Y}
$$

Similar to (7), the relation between the matrix $\mathbf{X}(\lambda x+\eta)$ and its derivative $\mathbf{X}^{\prime}(\lambda x+\eta)$

$$
\mathbf{X}^{\prime}(\lambda x+\eta)=\mathbf{X}(\lambda x+\eta) \mathbf{B}
$$

From (9) and (10) we obtain the another recurrence relation

$$
\begin{gathered}
{\left[y^{\prime}(\lambda x+\eta)\right]=\mathbf{X}^{\prime}(\lambda x+\eta) \mathbf{Y}=\mathbf{X}(\lambda x+\eta) \mathbf{B Y}} \\
{\left[y^{\prime \prime}(\lambda x+\eta)\right]=\mathbf{X}^{\prime}(\lambda x+\eta) \mathbf{B} \mathbf{Y}=\mathbf{X}(\lambda x+\eta) \mathbf{B}^{2} \mathbf{Y}} \\
\vdots \quad \vdots \\
{\left[y^{(k)}(\lambda x+\eta)\right]=\mathbf{X}^{\prime}(\lambda x+\eta) \mathbf{B}^{(k-1)} \mathbf{Y}=\mathbf{X}(\lambda x+\eta) \mathbf{B}^{k} \mathbf{Y}}
\end{gathered}
$$

Also the relation between the matrix $\mathbf{X}(x)$ and $\mathbf{X}(\lambda x+\eta)$

$$
\mathbf{X}(\lambda x+\eta)=\mathbf{X}(x) \mathbf{B}(\lambda, \eta)
$$

From (11) and (12) we can write

$$
\left[y^{(k)}(\lambda x+\eta)\right]=\mathbf{X}(x) \mathbf{B}(\lambda, \eta) \mathbf{B}^{k} \mathbf{Y}
$$

where for $\lambda \neq 0$ and $\eta \neq 0$

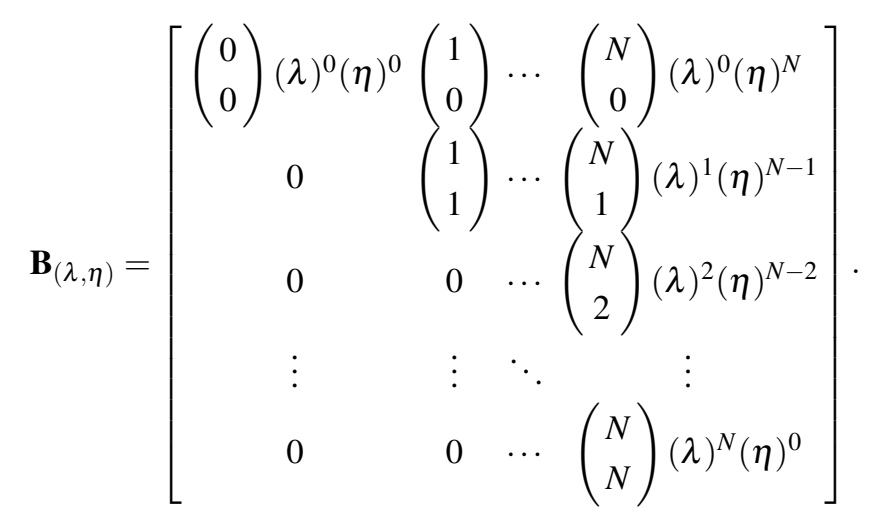

It is clear from (13) that

$$
\left[y^{(j)}\left(\eta_{i j} x+\tau_{i j}\right)\right]=\mathbf{X}(x) \mathbf{B}\left(\eta_{i j}, \tau_{i j}\right) \mathbf{B}^{j} \mathbf{Y}
$$

and

$$
\left[y^{(j)}(x-t)\right]=\mathbf{X}(x) \mathbf{B}(1,-t) \mathbf{B}^{j} \mathbf{Y}
$$

so that

$$
\mathbf{B}\left(\eta_{i j}, \tau_{i j}\right)=\left[b_{m, n}\right], \quad b_{m, n}=\left(\begin{array}{c}
n \\
m
\end{array}\right) \eta_{i j}^{m} \tau_{i j}^{n-m}, n<m \Rightarrow b_{m, n}=0 ; \quad m, n=0,1, \ldots, N
$$




$$
\mathbf{B}(1,-t)=\left[b_{i, j}\right], \quad b_{i, j}=\left(\begin{array}{c}
j \\
i
\end{array}\right)(-1)^{i+j} t^{j-i}, j<i \Rightarrow b_{i, j}=0 ; i, j=0,1, \ldots, N .
$$

To obtain the desired solution, we can use Taylor collocation method; therefore, we substitute collocation points (4) into Eq. (5) and obtain the system

$$
D\left(x_{s}\right)+F\left(x_{s}\right)+I\left(x_{s}\right)=g\left(x_{s}\right), \quad s=0,1, \ldots, N
$$

so that

$$
\begin{gathered}
D\left(x_{s}\right)=\sum_{i=0}^{m} p_{i}\left(x_{s}\right) y^{(i)}\left(x_{s}\right), F\left(x_{s}\right)=\sum_{i=0}^{m} \sum_{j=0}^{n} q_{i j}\left(x_{s}\right) y^{(i)}\left(\eta_{i j} x_{s}+\tau_{i j}\right), \\
I\left(x_{s}\right)=\sum_{j=0}^{n} \int_{a}^{b} K_{j}\left(x_{s}, t\right) y^{(j)}\left(x_{s}-t\right) d t
\end{gathered}
$$

then we can write the system (16) in the matrix form

$$
\mathbf{D}+\mathbf{F}+\mathbf{I}=\mathbf{G}
$$

where

$$
\mathbf{D}=\left[\begin{array}{c}
D\left(x_{0}\right) \\
D\left(x_{1}\right) \\
D\left(x_{2}\right) \\
\vdots \\
D\left(x_{N}\right)
\end{array}\right], \mathbf{F}=\left[\begin{array}{c}
F\left(x_{0}\right) \\
F\left(x_{1}\right) \\
F\left(x_{2}\right) \\
\vdots \\
F\left(x_{N}\right)
\end{array}\right], \mathbf{I}=\left[\begin{array}{c}
I\left(x_{0}\right) \\
I\left(x_{1}\right) \\
I\left(x_{2}\right) \\
\vdots \\
I\left(x_{N}\right)
\end{array}\right], \mathbf{G}=\left[\begin{array}{c}
g\left(x_{0}\right) \\
g\left(x_{1}\right) \\
g\left(x_{2}\right) \\
\vdots \\
g\left(x_{N}\right)
\end{array}\right]
$$

\section{A. Matrix representation of differential part}

To reduce the part $\mathbf{D}(x)$ to the matrix form by means of the collocation points (4) we first write the matrix $\mathbf{D}$ defined in Eq.(17) as

$$
\mathbf{D}=\sum_{i=0}^{m} \mathbf{P}_{i} \mathbf{Y}^{(i)}
$$

where

$$
\mathbf{P}_{i}=\left[\begin{array}{ccccc}
P_{i}\left(x_{0}\right) & 0 & 0 & \cdots & 0 \\
0 & P_{i}\left(x_{1}\right) & 0 & \cdots & 0 \\
0 & 0 & P_{i}\left(x_{2}\right) & \cdots & 0 \\
\vdots & \vdots & \vdots & \ddots & \vdots \\
0 & 0 & 0 & \cdots & P_{i}\left(x_{N}\right)
\end{array}\right], \mathbf{Y}^{(i)}=\left[\begin{array}{c}
y^{(i)}\left(x_{0}\right) \\
y^{(i)}\left(x_{1}\right) \\
y^{(i)}\left(x_{2}\right) \\
\vdots \\
y^{(i)}\left(x_{N}\right)
\end{array}\right]
$$

By substituting collocation points (4) into Eq. (8), we have the matrix equation

$$
\mathbf{Y}^{(i)}=\mathbf{X B}^{i} \mathbf{Y}
$$

where

$$
\mathbf{X}=\left[\begin{array}{c}
X\left(x_{0}\right) \\
X\left(x_{1}\right) \\
X\left(x_{2}\right) \\
\vdots \\
X\left(x_{N}\right)
\end{array}\right]=\left[\begin{array}{ccccc}
1 & \left(x_{0}-c\right) & \left(x_{0}-c\right)^{2} & \cdots & \left(x_{0}-c\right)^{N} \\
1 & \left(x_{1}-c\right) & \left(x_{1}-c\right)^{2} & \cdots & \left(x_{1}-c\right)^{N} \\
1 & \left(x_{2}-c\right) & \left(x_{2}-c\right)^{2} & \cdots & \left(x_{2}-c\right)^{N} \\
\vdots & \vdots & \vdots & \ddots & \vdots \\
1 & \left(x_{N}-c\right) & \left(x_{N}-c\right)^{2} & \cdots & \left(x_{N}-c\right)^{N}
\end{array}\right]
$$


Consequently, from the matrix forms (18) and (19), we obtain the fundamental matrix relation for the differential part $D(x)$

$$
\mathbf{D}=\sum_{i=1}^{m} \mathbf{P}_{i} \mathbf{X B}^{i} \mathbf{Y}
$$

\section{B. Matrix representation of differential-difference part}

Let us now find the matrix $\mathbf{F}$ corresponding to the part $\mathbf{F}(x)$. From (14) we can write $\mathbf{F}(x)$ in matrix form

$$
[F(x)]=\sum_{i=0}^{m} \sum_{j=0}^{n} q_{i j} \mathbf{X}(x) \mathbf{B}\left(\eta_{i j}, \tau_{i j}\right) \mathbf{B}^{i} \mathbf{Y}
$$

for $x=x_{s}$, we obtain the matrix system, from (21),

$$
\mathbf{F}=\sum_{i=0}^{m} \sum_{j=0}^{n} \mathbf{Q}_{i j} \mathbf{X B}\left(\eta_{i j}, \tau_{i j}\right) \mathbf{B}^{i} \mathbf{Y}
$$

where

$$
\mathbf{Q}_{i j}=\left[\begin{array}{ccccc}
q_{i j}\left(x_{0}\right) & 0 & 0 & \cdots & 0 \\
0 & q_{i j}\left(x_{1}\right) & 0 & \cdots & 0 \\
0 & 0 & q_{i j}\left(x_{2}\right) & \cdots & 0 \\
\vdots & \vdots & \vdots & \ddots & \vdots \\
0 & 0 & 0 & \cdots & q_{i j}\left(x_{N}\right)
\end{array}\right], i=0,1, \ldots, m ; j=0,1, \ldots, n
$$

\section{Matrix representation of integral part}

Now we find the matrix I corresponding to the part $\mathbf{I}(x)$. The kernel $K_{j}(x, t)$ can be expanded to the truncated Taylor series in the form

$$
K_{j}(x, t)=\sum_{r=0}^{N} \sum_{s=0}^{N} k_{r s}^{j}(x-c)^{r}(t-c)^{s}
$$

where

$$
k_{r s}^{j}=\frac{1}{r ! s !} \frac{\partial^{r+s} K_{j}(c, c)}{\partial x^{r} \partial t^{s}}, r, s=0,1, \ldots, N ; j=0,1, \ldots, N
$$

then the matrix representation of $K_{j}(x, t)$ can be given as

$$
\left[K_{j}(x, t)\right]=\mathbf{X}(x) \mathbf{K}_{j} \mathbf{X}^{T}(t)
$$

where

$$
\begin{gathered}
\mathbf{K}_{j}=\left[k_{r s}^{j}\right] ; r, s=0,1, \ldots, N ; j=0,1, \ldots, N \\
K_{j}(x, t)=\sum_{r=0}^{N} \sum_{s=0}^{N} k_{r s}^{j}(x-c)^{r}(t-c)^{s} \\
\mathbf{X}(t)=\left[1(t-c)(t-c)^{2} \cdots(t-c)^{N}\right]
\end{gathered}
$$


Substituting the matrix forms (15) and (23) corresponding to the functions $y^{(j)}(x-t)$ and $\mathbf{K}_{j}(x, t)$ into the integral part $\mathbf{I}(x)$, we get the matrix

$$
\begin{aligned}
\mathbf{I}(x) & =\sum_{j=0}^{n} \int_{a}^{b} \mathbf{X}(x) \mathbf{K}_{j} \mathbf{X}^{T}(t) \mathbf{X}(x) \mathbf{B}(1,-t) \mathbf{B}^{j} \mathbf{Y} d t \\
& =\sum_{j=0}^{N} \mathbf{X}(x) \mathbf{K}_{j}\left\{\int_{a}^{b} \mathbf{X}^{T}(t) \mathbf{X}(x) \mathbf{B}(1,-t) d t\right\} \mathbf{B}^{j} \mathbf{Y}
\end{aligned}
$$

in order to make easy the calculation of $\mathbf{I}(x)$, we use the next equation

$$
\mathbf{X}^{T}(x) \mathbf{X}(x)=\widetilde{\mathbf{X}}(x) \mathbf{H}(t)
$$

then we find the matrix relation

$$
\begin{aligned}
& \mathbf{I}(x)=\sum_{j=0}^{n} \int_{a}^{b} \mathbf{X}(x) \mathbf{K}_{j} \mathbf{X}^{T}(t) \mathbf{X}(x) \mathbf{B}(1,-t) \mathbf{B}^{j} \mathbf{Y} d t \\
& =\sum_{j=0}^{N} \mathbf{X}(x) \mathbf{K}_{j} \widetilde{\mathbf{X}}(x) \mathbf{B}(1,-t)\left\{\int_{a}^{b} \mathbf{H}(t) \mathbf{B}(1,-t) d t\right\} \mathbf{B}^{j} \mathbf{Y}
\end{aligned}
$$

where

$$
\mathbf{H}(t)=\left[\begin{array}{c}
\mathbf{I} \\
(t-c) \mathbf{I} \\
(t-c)^{2} \mathbf{I} \\
\vdots \\
(t-c)^{N} \mathbf{I}
\end{array}\right], \widetilde{\mathbf{X}}(x)=\left[\begin{array}{ccccc}
\mathbf{X} & 0 & 0 & \cdots & 0 \\
0 & \mathbf{X} & 0 & \cdots & 0 \\
0 & 0 & \mathbf{X} & \cdots & 0 \\
\vdots & \vdots & \vdots & \ddots & \vdots \\
0 & 0 & 0 & \cdots & \mathbf{X}
\end{array}\right]
$$

here $\mathbf{I}$ is the $(N+1) x(N+1)$ matrix. By putting the collocation points $x_{s}, s=0,1, \ldots, N$ defined in (4) in the relation (24) we obtain the system of matrix equations

$$
\begin{aligned}
& \mathbf{I}\left(x_{s}\right)=\sum_{j=0}^{n} \int_{a}^{b} \mathbf{X}\left(x_{s}\right) \mathbf{K}_{j} \mathbf{X}^{T}(t) \mathbf{X}\left(x_{s}\right) \mathbf{B}(1,-t) \mathbf{B}^{j} \mathbf{Y} d t \\
& =\sum_{j=0}^{N} \mathbf{X}\left(x_{s}\right) \mathbf{K}_{j} \widetilde{\mathbf{X}}\left(x_{s}\right) \mathbf{B}(1,-t)\left\{\int_{a}^{b} \mathbf{H}(t) \mathbf{B}(1,-t) d t\right\} \mathbf{B}^{j} \mathbf{Y}
\end{aligned}
$$

or briefly the matrix relation

$$
\mathbf{I}=\sum_{j=0}^{N} \mathbf{X}^{*}(x) \mathbf{K}_{j}^{*} \widetilde{\mathbf{X}} \mathbf{A} \mathbf{B}^{j} \mathbf{Y}
$$

which is the fundamental matrix relation for the integral part $\mathbf{I}(x)$. Here

$$
\mathbf{A}=\int_{a}^{b} \mathbf{H}(t) \mathbf{B}(1,-t) d t=\left[\begin{array}{c}
\int_{a}^{b} \mathbf{B}(1,-t) d t \\
\int_{a}^{b}(t-c) \mathbf{B}(1,-t) d t \\
\int_{a}^{b}(t-c)^{2} \mathbf{B}(1,-t) d t \\
\vdots \\
\int_{a}^{b}(t-c)^{N} \mathbf{B}(1,-t) d t
\end{array}\right]=\left[\begin{array}{c}
\mathbf{A}_{0} \\
\mathbf{A}_{1} \\
\mathbf{A}_{2} \\
\vdots \\
\mathbf{A}_{N}
\end{array}\right], \widetilde{\mathbf{X}}=\left[\begin{array}{c}
\widetilde{\mathbf{X}}\left(x_{0}\right) \\
\widetilde{\mathbf{X}}\left(x_{1}\right) \\
\widetilde{\mathbf{X}}\left(x_{2}\right) \\
\vdots \\
\widetilde{\mathbf{X}}\left(x_{N}\right)
\end{array}\right]
$$


for $A_{k}, k=0,1,2, \ldots, N$ we can write general formula

$$
\begin{gathered}
\mathbf{A}=\left[a_{i j}^{k}\right]=\left(\begin{array}{c}
j \\
i
\end{array}\right)(-1)^{i+j}\left\{\frac{b^{j-i+k+1}-a^{j-i+k+1}}{j-i+k+1}\right\}, \\
j<i \Rightarrow a_{i j}^{k}=0 ; i, j, k=0,1,2, \ldots, N
\end{gathered}
$$

and

$$
\mathbf{X}^{*}=\left[\begin{array}{ccccc}
\mathbf{X}\left(x_{0}\right) & 0 & 0 & \cdots & 0 \\
0 & \mathbf{X}\left(x_{1}\right) & 0 & \cdots & 0 \\
0 & 0 & \mathbf{X}\left(x_{2}\right) & \cdots & 0 \\
\vdots & \vdots & \vdots & \ddots & \vdots \\
0 & 0 & 0 & \cdots & \mathbf{X}\left(x_{N}\right)
\end{array}\right], \mathbf{K}_{j}^{*}=\left[\begin{array}{ccccc}
\mathbf{K}_{j} & 0 & 0 & \cdots & 0 \\
0 & \mathbf{K}_{j} & 0 & \cdots & 0 \\
0 & 0 & \mathbf{K}_{j} & \cdots & 0 \\
\vdots & \vdots & \vdots & \ddots & \vdots \\
0 & 0 & 0 & \cdots & \mathbf{K}_{j}
\end{array}\right]
$$

\section{Matrix relation for the mixed conditions}

We can obtain the corresponding matrix form for the conditions (2) as follow. By using the recurrence relations (8) for $y^{(j)}(a), y^{(j)}(b)$, and $y^{(j)}(c), j=0,1,2, \ldots, m-1$ we have matrix equations of (2)

$$
\sum_{j=0}^{m-1}\left[a_{i j} \mathbf{X}(a)+b_{i j} \mathbf{X}(b)+c_{i j} \mathbf{X}(c)\right] \mathbf{B}^{j} \mathbf{Y}=\left[\mu_{i}\right], \quad i=0,1,2, \ldots, m-1, a \leq b \leq c
$$

briefly, the matrix forms for conditions (2) are

$$
\mathbf{U}_{i} \mathbf{Y}=\left[\mu_{i}\right] ; \quad i=0,1,2, \ldots, m-1
$$

where

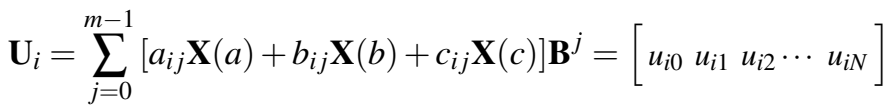

\section{Method of solution}

We now ready to construct the fundamental matrix equation corresponding to Eq.(1) for this purpose, we first substitute (20),(22) and (25) ) into Eq.(1) and then simplify. Thus we have the fundamental matrix equation

$$
\left\{\sum_{i=1}^{m} \mathbf{P}_{i} \mathbf{X}(x) \mathbf{B}^{i}+\sum_{i=0}^{m} \sum_{j=0}^{n} \mathbf{Q}_{i j} \mathbf{X}(x) \mathbf{B}\left(\eta_{i j}, \tau_{i j}\right) \mathbf{B}^{i}+\sum_{j=0}^{N} \mathbf{X}^{*} \mathbf{K}_{j}^{*} \widetilde{\mathbf{X}} \mathbf{A} \mathbf{B}^{j} \mathbf{Y}\right\} \mathbf{Y}=\mathbf{G}
$$

which corresponds to a system of $N+1$ algebraic equations for the $N+1$ unknown Taylor coefficients $y_{0}, y_{1}, y_{2}, \ldots, y_{N}$. Briefly, Eq.(26) can be written in the form

$$
\mathbf{W Y}=\mathbf{G} \text { or }[\mathbf{W} ; \mathbf{G}]
$$

so that

$$
\begin{gathered}
\mathbf{W}=\left[w_{p q}\right]=\sum_{i=1}^{m} \mathbf{P}_{i} \mathbf{X} \mathbf{B}^{i}+\sum_{i=0}^{m} \sum_{j=0}^{n} \mathbf{Q}_{i j} \mathbf{X}(x) \mathbf{B}\left(\eta_{i j}, \tau_{i j}\right) \mathbf{B}^{i}+\sum_{j=0}^{n} \mathbf{X}^{*} \mathbf{K}_{j}^{*} \widetilde{\mathbf{X}} \mathbf{A B}^{j}, \\
p, q=0,1,2, \ldots, N
\end{gathered}
$$


the augmented matrix of Eq.(27) becomes

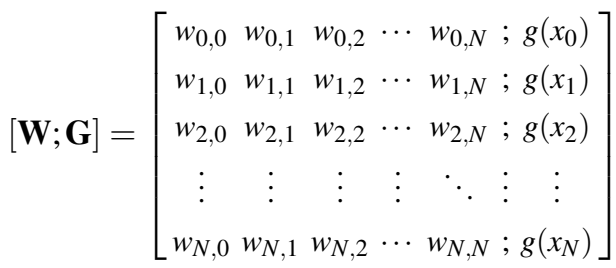

We now consider the matrix Eq.(28) corresponding to the conditions (2). Then the augmented matrix of Eq.(2) becomes

$$
\left[\mathbf{U}_{i}, \mu_{i}\right]=\left[\begin{array}{lllll}
u_{i 0} & u_{i 1} & u_{i 2} & \cdots & u_{i N} ; \mu_{i}
\end{array}\right], i=0,1,2, \ldots, m-1
$$

To obtain the solution of Eq.(1) under conditions (2), the following augmented matrix is constructed by replacing the row matrices (28) by the last m rows of the matrix (29) so we have new augmented matrix

$$
\widetilde{\mathbf{W}} ; \widetilde{\mathbf{G}}]=\left[\begin{array}{ccccccc}
w_{0,0} & w_{0,1} & w_{0,2} & \cdots & w_{0, N} & ; & g\left(x_{0}\right) \\
w_{1,0} & w_{1,1} & w_{1,2} & \cdots & w_{1, N} & ; & g\left(x_{1}\right) \\
w_{2,0} & w_{2,1} & w_{2,2} & \cdots & w_{2, N} & ; & g\left(x_{2}\right) \\
\vdots & \vdots & \vdots & \vdots & \ddots & \vdots & \vdots \\
w_{N-m, 0} & w_{N-m, 1} & w_{N-m, 2} & \cdots & w_{N-m, N} & ; g\left(x_{N-m}\right) \\
u_{0,0} & u_{0,1} & u_{0,2} & \cdots & u_{0, N} & ; & \mu_{0} \\
u_{1,0} & u_{1,1} & u_{1,2} & \cdots & u_{1, N} & ; & \mu_{1} \\
u_{2,0} & u_{2,1} & u_{2,2} & \cdots & u_{2, N} & ; & \mu_{2} \\
\vdots & \vdots & \vdots & \vdots & \ddots & \vdots & \vdots \\
u_{m-1,0} & u_{m-1,1} & u_{m-1,2} & \cdots & u_{m-1, N} & ; & \mu_{m-1}
\end{array}\right]
$$

from this linear system, the unknown Taylor coefficients are determined and substituted in (3); thus we get the Taylor polynomial solution

$$
y(x)=\sum_{n=0}^{N} y_{n}(x-c)^{n}, \quad y_{n}=\frac{y^{(n)}(c)}{n !}, a \leq x, c \leq b
$$

\section{Accuracy of the solution and error analysis}

We can easily check the accuracy of the method. Since the truncated Taylor series (3) is an approximate solution of Eq. (1), when the function $y_{N}(x)$ and its derivatives are substituted in Eq. (1), the resulting equation must be satisfied approximately; that is, for $x=x_{s} \in[a, b] s=0,1,2, \ldots, N$

$$
E\left(x_{s}\right)=\left|D\left(x_{s}\right)-F\left(x_{s}\right)-I\left(x_{s}\right)-g\left(x_{s}\right)\right| \cong 0
$$

and $E\left(x_{s}\right) \leq 10^{-k i}$ (-ki positive integer) If $\max 10^{-k i}=10^{-k}$ ( $k$ positive integer) is prescribed, then the truncation limit $N$ is increased until the difference $E\left(x_{i}\right)$ at each of the points becomes smaller than the prescribed $10^{-k}$. On the other hand, the error can be estimated by the function

$$
E_{N}(x)=\sum_{i=0}^{m} p_{i}(x) y_{N}^{(i)}(x)-F(x)-I(x)-g(x),
$$


if $E_{N}(x) \rightarrow 0$ when $N$ is sufficiently large enough, then the error decreases.

\section{Numerical examples}

The method of this study is useful in finding the solutions of mixed linear integro-differential-difference equations in terms of Taylor polynomials. We illustrate it by the following examples.

Example 4.1 Let us consider the problem

$$
\begin{gathered}
(x-2) y^{\prime \prime}(x)+x y(x-1)+\int_{a}^{b} x y^{\prime}(x-t) d t=\left(x^{2}-1\right) e^{x}+x e^{x}, \\
y(0)=1, \quad y^{\prime}(0)=1, \quad 0 \leq x \leq 1
\end{gathered}
$$

and approximate the solution $y_{N}(x)$ by the truncated Taylor series in the form

$$
y(x)=\sum_{n=0}^{N} y_{n}(x-c)^{n}, \quad 0 \leq x \leq 1
$$

so that $N=5, p_{2}(x)=\left(x^{2}-1\right), q_{00}=x, K_{1}(x, t)=x, g(x)=\left(x^{2}-1\right) e^{x}+x e^{x}$ For $N=5$ Taylor collocation points are

$$
\begin{gathered}
x_{i}=a+\frac{b-a}{N} i=0+\frac{i}{N}=\frac{i}{5} ; \\
i=0,1, \ldots, 5 \Rightarrow x_{0}=0, x_{1}=\frac{1}{5}, x_{2}=\frac{2}{5}, x_{3}=\frac{3}{5}, x_{4}=\frac{4}{5}, x_{5}=1 .
\end{gathered}
$$

The matrix representation of the equation is

$$
\left\{\mathbf{P}_{2} \mathbf{X B}^{2}+\mathbf{Q}_{00} \mathbf{X B}_{1,-1}+\mathbf{X}^{*} \mathbf{K}_{1}^{*} \widetilde{\mathbf{X}} \mathbf{A B}\right\} \mathbf{Y}=\mathbf{G}
$$

simplifying the result, we have the augmented matrix

$$
[\widetilde{\mathbf{W}} ; \widetilde{\mathbf{G}}]=\left[\begin{array}{cccccccc}
0 & 0 & -2 & 0 & 0 & 0 & ; & -1 \\
0.2 & 0.04 & -1.9120 & -1.1504 & -0.4605 & -0.1535 & ; & -0.9283 \\
0.4 & 0.16 & -1.6160 & -1.9904 & -1.6026 & -1.0711 & ; & -0.6564 \\
0.6 & 0.36 & 0.36 & -1.0640 & -2.1744 & -2.6870 & ; & -2.7181 \\
1 & 0 & 0 & 0 & 0 & 0 & ; & 1 \\
0 & 1 & 0 & 0 & 0 & 0 & ; & 1
\end{array}\right] .
$$

The solution of this system gives the Taylor coefficients

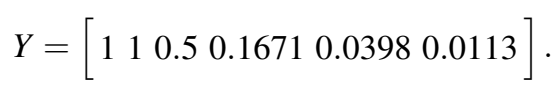

Thus, the solution of this problem becomes

$$
y_{5}(x)=1+x+\frac{1}{2} x^{2}+0.1671 x^{3}+0.0398 x^{4}+0.0113 x^{5} .
$$

The exact solution of this problem is $y=e^{x}$ and the comparison of approximate solutions (for $N=5,7,9$ ) with exact solution is given in Table 1. and Fig. 1. 
Table 1. Comparison of the absolute errors of Example 1.

\begin{tabular}{ccccc}
\hline \hline \multicolumn{5}{c}{ Error analysis $\left(\left|y-y^{*}\right|\right)$} \\
$x_{r}$ & Exact Solution & $N=5$ & $N=7$ & $N=9$ \\
\hline 0.0 & 1.000000000 & 0.00000 & 0.00000 & 0.00000 \\
0.1 & 1.105170918 & $2.64 \mathrm{E}-7$ & $1.00 \mathrm{E}-9$ & 0.00000 \\
0.2 & 1.221402758 & $1.21 \mathrm{E}-6$ & $3.00 \mathrm{E}-9$ & 0.00000 \\
0.3 & 1.349858808 & $2.19 \mathrm{E}-6$ & $5.00 \mathrm{E}-9$ & 0.00000 \\
0.4 & 1.491824698 & $1.37 \mathrm{E}-5$ & $1.00 \mathrm{E}-10$ & 0.00000 \\
0.5 & 1.648721271 & $2.75 \mathrm{E}-6$ & $7.00 \mathrm{E}-9$ & 0.00000 \\
0.6 & 1.822118800 & $4.76 \mathrm{E}-6$ & $9.00 \mathrm{E}-10$ & 0.00000 \\
0.7 & 2.013752707 & $5.74 \mathrm{E}-6$ & $1.00 \mathrm{E}-8$ & 0.00000 \\
0.8 & 2.225540928 & $5.58 \mathrm{E}-7$ & $1.41 \mathrm{E}-8$ & 0.00000 \\
0.9 & 2.459603111 & $3.23 \mathrm{E}-5$ & $5.51 \mathrm{E}-8$ & $1.00 \mathrm{E}-9$ \\
1.0 & 2.718281828 & $1.26 \mathrm{E}-4$ & $3.81 \mathrm{E}-8$ & $3.00 \mathrm{E}-9$ \\
\hline \hline
\end{tabular}

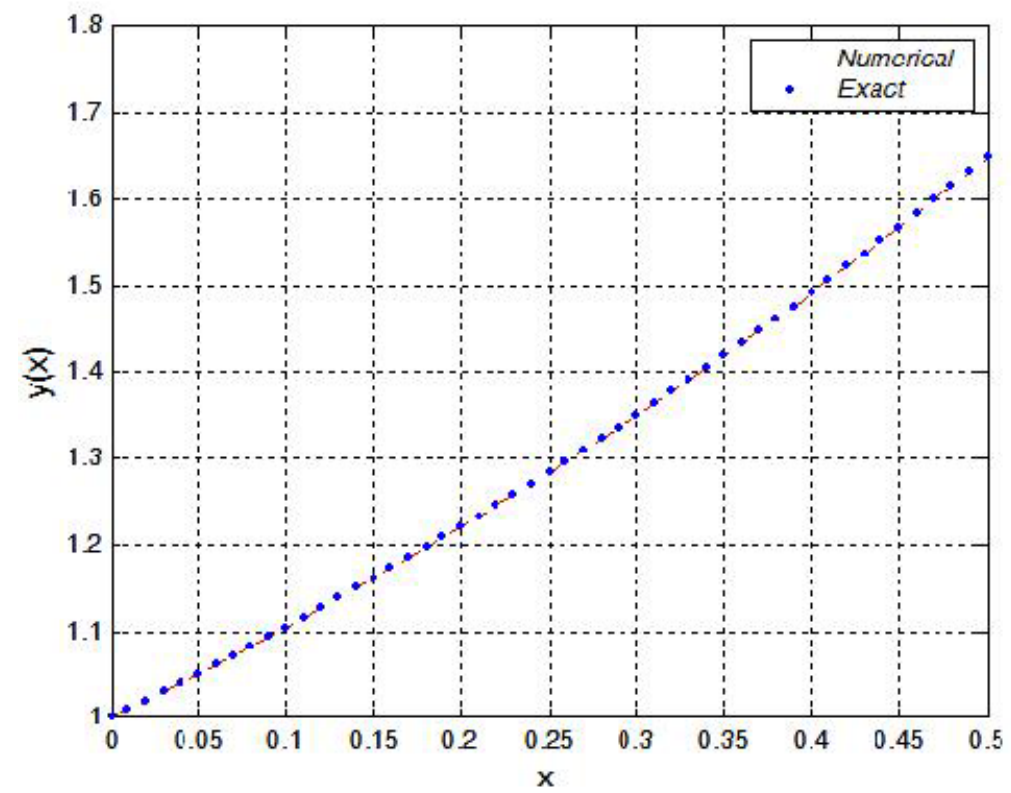

Fig. 1. Exact and numerical results for Example 1

Example 4.2 Consider the multi-pantograph equation

$$
y^{\prime}(x)=-y(x)-e^{-0.5 x} \sin (0.5 x)+y(0.5 x)-2 e^{-0.75 x} \cos (0.5 x) \sin (0.25 x) y(0.25 x), 0 \leq x \leq 1
$$


with initial conditions $y(0)=1$ and its exact solution is $y(x)=e^{-x} \cos (x)$ [15]. Following the procedure in the previous example we get the approximate solution by the Taylor polynomial for $N=5$ as

$$
y_{5}(x)=1-x+0.2699984508 e-3 x^{2}+0.3314703344 x^{3}-0.1621004024 x^{4}+0.2917391544 e-5 x^{5}
$$

In Table 2 we compare the absolute errors of the present method for $N=5,7,9$ Fig. 2. shows the comparison of error functions.

Table 2. Comparison of the absolute errors of Example 2.

\begin{tabular}{ccccc}
\hline \hline \multicolumn{5}{c}{ Error analysis $\left(\left|y-y^{*}\right|\right)$} \\
$x_{r}$ & Exact Solution & $N=6$ & $N=8$ & $N=10$ \\
\hline 0.0 & 1.000000000 & 0.00000 & 0.00000 & 0.00000 \\
0.1 & 0.9003169998 & $1.18 \mathrm{E}-7$ & $7.00 \mathrm{E}-10$ & 0.00000 \\
0.2 & 0.8024106473 & $3.23 \mathrm{E}-7$ & $6.00 \mathrm{E}-10$ & $2.15 \mathrm{E}-10$ \\
0.3 & 0.7077306780 & $4.19 \mathrm{E}-8$ & $6.00 \mathrm{E}-10$ & $1.10 \mathrm{E}-10$ \\
0.4 & 0.6174056479 & $1.48 \mathrm{E}-8$ & $6.00 \mathrm{E}-10$ & $1.17 \mathrm{E}-10$ \\
0.5 & 0.5322807302 & $3.52 \mathrm{E}-8$ & $5.00 \mathrm{E}-10$ & 0.00000 \\
0.6 & 0.4529537891 & $1.30 \mathrm{E}-8$ & $5.00 \mathrm{E}-10$ & 0.00000 \\
0.7 & 0.3798093899 & $3.15 \mathrm{E}-8$ & $1.00 \mathrm{E}-10$ & 0.00000 \\
0.8 & 0.3130505040 & $1.67 \mathrm{E}-7$ & $2.41 \mathrm{E}-10$ & $1.10 \mathrm{E}-10$ \\
0.9 & 0.2527277533 & $7.29 \mathrm{E}-8$ & $5.55 \mathrm{E}-10$ & $3.10 \mathrm{E}-10$ \\
1.0 & 0.1987661104 & $6.20 \mathrm{E}-6$ & $3.12 \mathrm{E}-8$ & $5.18 \mathrm{E}-8$ \\
\hline \hline
\end{tabular}

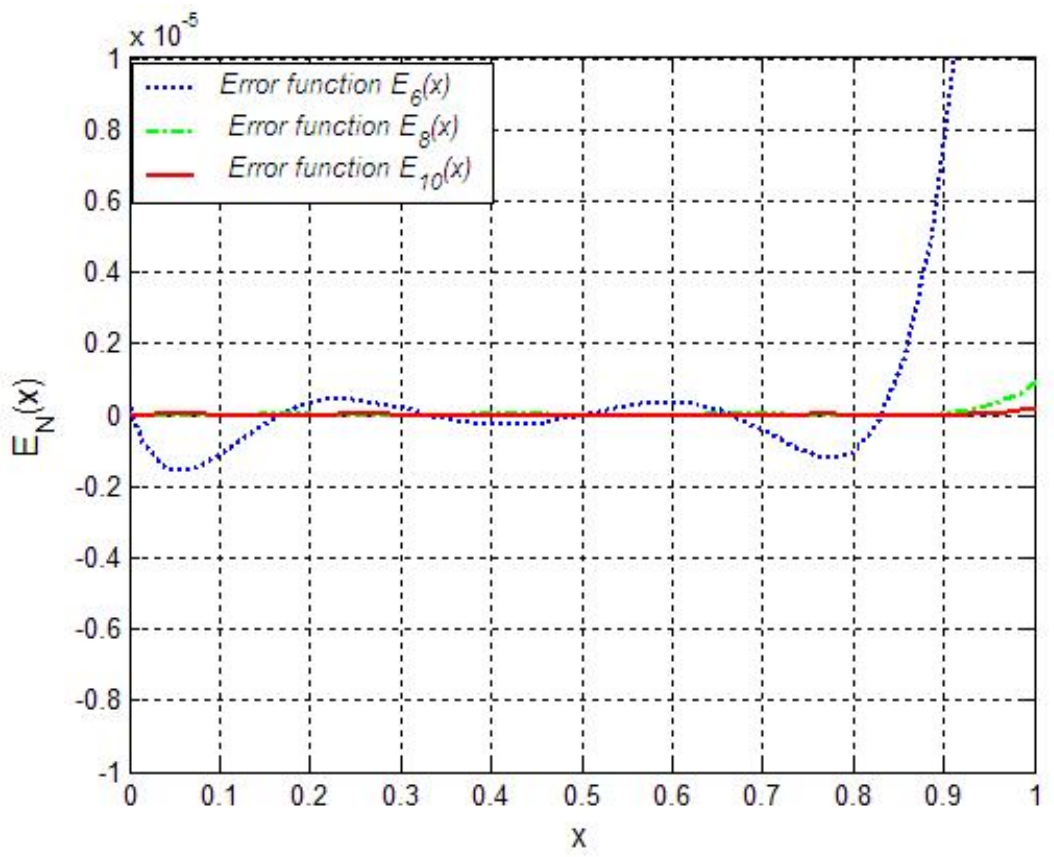

Fig. 2. Comparison of error functions of Example 2 
Example 4.3 [3]. Consider the difference equation

$$
\begin{gathered}
(x-1) y(x+2)+(2-3 x) y(x+1)+2 x y(x)=1, \\
y(0)=2, y(1)=2,0 \leq x \leq 2
\end{gathered}
$$

The exact solution is $y(x)=2^{x}-x+1$. For numerical result, see Table 3 and Fig. 3 .

Table 3. Comparison of the absolute errors of Example 3

\begin{tabular}{ccccc}
\hline \hline \multicolumn{5}{c}{ Error analysis $\left(\left|y-y^{*}\right|\right)$} \\
$x_{r}$ & Exact Solution & $N=6$ & $N=8$ & $N=10$ \\
\hline 0.0 & 2.000000000 & 0.00000 & 0.00000 & 0.00000 \\
-0.4 & 2.157858283 & $5.63 \mathrm{E}-6$ & $1.23 \mathrm{E}-7$ & $2.12 \mathrm{E}-9$ \\
-0.8 & 2.374349178 & $2.03 \mathrm{E}-5$ & $1.24 \mathrm{E}-7$ & $2.10 \mathrm{E}-9$ \\
-0.12 & 2.635275282 & $1.54 \mathrm{E}-9$ & $2.00 \mathrm{E}-9$ & $3.17 \mathrm{E}-9$ \\
-1.16 & 2.929876978 & $5.10 \mathrm{E}-5$ & $1.07 \mathrm{E}-7$ & $5.28 \mathrm{E}-8$ \\
-2 & 3.250000000 & $2.68 \mathrm{E}-3$ & $2.11 \mathrm{E}-5$ & $1.05 \mathrm{E}-7$ \\
\hline \hline
\end{tabular}

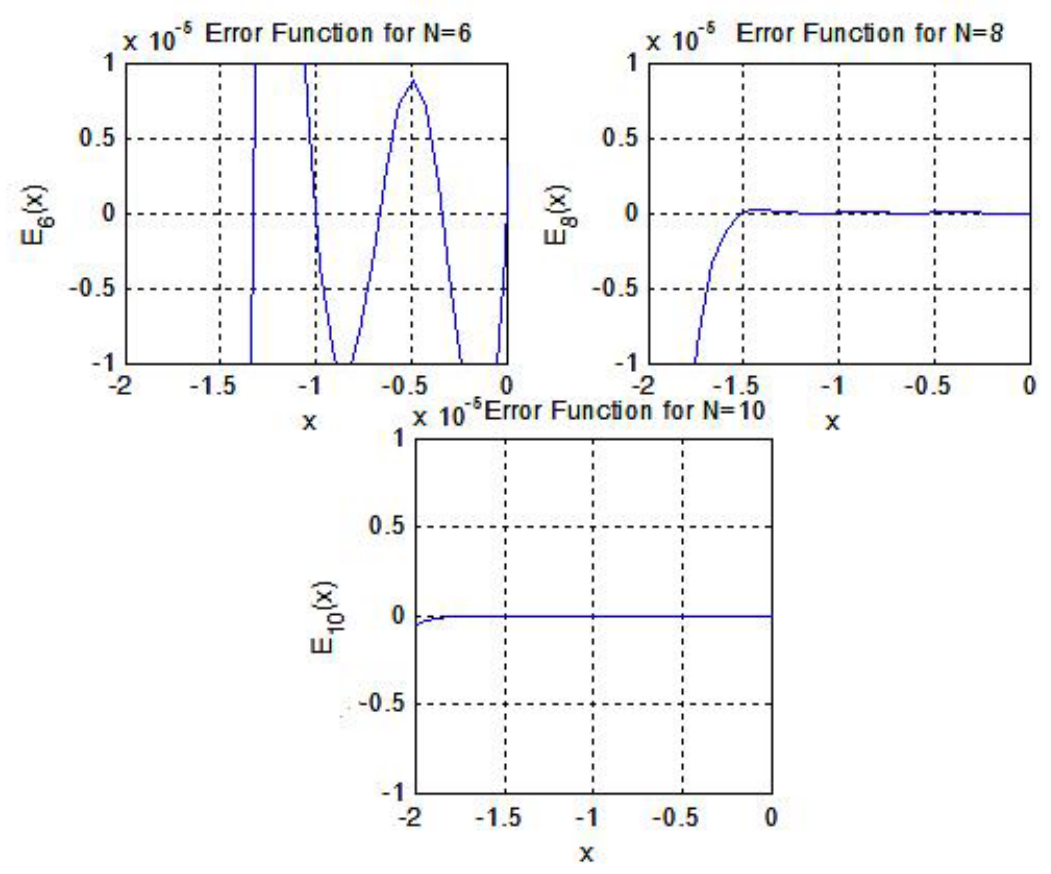

Fig. 3. Comparison of error functions of Example 3

Example 4.4 In this example, we consider the linear delay differential equation of third order [12]

$$
\frac{d^{3} y}{d x^{3}}=-y(x)-y(x-0.3)+e^{-x+0.3}
$$

and the following initial conditions

$$
y(0)=1, y^{\prime}(0)=-1, y^{\prime \prime}(0)=1
$$


with the exact solution $y(x)=e^{-x}$ Table 4 compares the results of the present method and the differential transform method.

Table 4. Error analysis of Example 4

\begin{tabular}{ccccc}
\hline \hline \multicolumn{4}{c}{ Present Method $\left(\left|y-y^{*}\right|\right)$} \\
$x_{r}$ & $N=5$ Error & $N=8$ Error & $N=10$ Error & Differential Transform[14] \\
\hline 0.0 & 0.00000 & 0.00000 & 0.00000 & 0.00000 \\
0.1 & $3.32 \mathrm{E}-8$ & $6.57 \mathrm{E}-12$ & $7.68 \mathrm{E}-14$ & $6.40 \mathrm{E}-11$ \\
0.2 & $3.69 \mathrm{E}-7$ & $7.01 \mathrm{E}-11$ & $7.34 \mathrm{E}-14$ & $1.20 \mathrm{E}-10$ \\
0.3 & $1.21 \mathrm{E}-6$ & $6.99 \mathrm{E}-10$ & $1.66 \mathrm{E}-13$ & $8.17 \mathrm{E}-11$ \\
0.4 & $2.37 \mathrm{E}-6$ & $1.90 \mathrm{E}-10$ & $8.12-\mathrm{E} 12$ & $6.43 \mathrm{E}-11$ \\
0.5 & $3.64 \mathrm{E}-6$ & $2.53 \mathrm{E}-10$ & $1.71 \mathrm{E}-12$ & $8.73 \mathrm{E}-11$ \\
0.6 & $5.96 \mathrm{E}-6$ & $2.15 \mathrm{E}-10$ & $2.11 \mathrm{E}-12$ & $5.97 \mathrm{E}-12$ \\
0.7 & $1.32 \mathrm{E}-5$ & $1.34 \mathrm{E}-10$ & $1.81 \mathrm{E}-12$ & $2.08 \mathrm{E}-10$ \\
0.8 & $3.48 \mathrm{E}-5$ & $6.62 \mathrm{E}-9$ & $1.18 \mathrm{E}-12$ & $1.72 \mathrm{E}-11$ \\
0.9 & $2.96 \mathrm{E}-4$ & $2.74 \mathrm{E}-8$ & $6.30 \mathrm{E}-11$ & $1.59 \mathrm{E}-10$ \\
\hline \hline
\end{tabular}

Example 4.5 Finally, we consider the following neutral integro-differential equation

$$
\frac{d}{d x}[y(x)-3 y(x-1)]+\int_{0}^{1}(x-t) y(x-t) d t=\frac{-13}{6}+x^{2}
$$

with condition $y^{\prime}(0)=1$. Following the previous procedures, we get the approximate solution of problem for $N=5$ as $y(x)=x+1$ which is the exact solution.

\section{Conclusion}

The numerical results show that the accuracy improves when $N$ is increased. Tables and Figures indicate that as $N$ increases, the errors decrease more rapidly; hence for better results, using large number $N$ is recommended. A considerable advantage of the method is that Taylor coefficients of the solution are found very easily by using the computer programs. On the other hand our $N$ th order approximation gives the exact solution when the solution is polynomial of degree equal to or less than $N$. If the solution is not polynomial, Taylor series approximation converges to the exact solution as $N$ increases.

\section{References}

[1] S. Yalçınbaş, Taylor polynomial solutions of nonlinear Volterra-Fredholm integral equations, Appl. Math. Comput. 127 (2002)196206.

[2] M. Gülsu, M. Sezer, On the solution of the Riccati equation by the Taylor matrix method, Appl. Math. Comput. 188 (2007) 446-449.

[3] M. Sezer, Taylor polynomial solution of Volterra integral equations, Int. J. Math. Educ. Sci. Technol. 25 (5) (1994) $625-633$. 
[4] M. Sezer, M. Gülsu, A new polynomial approach for solving difference and Fredholm integro-difference equations with mixed argument, Appl. Math. Comput. 171 (2005) 332-344.

[5] S. Yalçınbaş, M. Sezer, The approximate solution of high-order linear Volterra-Fredholm integro-differential equations in terms of Taylor polynomials, Appl. Math. Comput. 112 (2000) 291-308.

[6] M. Sezer, A method for approximate solution of the second order linear differential equations in terms of Taylor polynomials, Int. J. Math. Educ. Sci. Technol. 27 (6) (1996) 821-834.

[7] Ş. Nas, S. Yalçınbaş, M. Sezer, A method for approximate solution of the high-order linear Fredholm integro-differential equations, Int. J. Math. Educ. Sci. Technol. 27 (6) (1996) 821-834.

[8] A. Karamete, M. Sezer, A Taylor collocation method for the solution of linear integro-differential equations, Int. J. Comput. Math. 79 (9) (2002) 987-1000.

[9] M. Gülsu, M. Sezer, A method for the approximate solution of the high-order linear difference equations in terms of Taylor polynomials, Int. J. Comput. Math 82 (5) (2005) 629-642.

[10] B. Bülbül, M. Gülsu, M. Sezer, A new Taylor collocation method for nonlinear Fredholm-Volterra integro-differential equations, Numer. Methods Partial Diff. Eq. doi 10.1002/num.20470.

[11] T. L. Saaty Modern Nonlinear Equations, Dover Publications Inc., New York, 1981.

[12] F. Karakoç, H. Bereketoğlu, Solutions of delay differential equations by using differential Transform method, Int. J. Comput. Math. 86 (2009) 914-923.

[13] M. Sezer, S. Yalçınbaş, N. Şahin, Approximate solution of multi-pantograph equation with variable coefficients, J. Comput. Appl. Math. 214 (2008) 406-416.

[14] B. Gürbüz, M. Gülsu, M. Sezer, Numerical approach of high-order linear delay difference equations with variable coefficients in terms of Laguerre polynomials, Math. Comput. Appl., 16 (2011) 267-278.

[15] B. Gürbüz, M. Sezer, Laguerre collocation method for solving Fredholm integro-differential equations with functional arguments, J. Appl. Math. 2014 (2014) 12. 